\title{
Communication \\ High-Responsivity, Low-Leakage Current, Ultra-Fast Terahertz Detectors Based on a GaN High-Electron-Mobility Transistor with Integrated Bowtie Antennas
}

\author{
Zhen Huang ${ }^{1,2,3} \mathbb{D}$, Wei Yan ${ }^{1, *}$, Zhaofeng Li ${ }^{1,2,4} \mathbb{D}$, Hui Dong ${ }^{1,5}$, Fuhua Yang ${ }^{2,4,6}$ and Xiaodong Wang ${ }^{1,2,4,7,8} \mathbb{D}$ \\ 1 Engineering Research Center for Semiconductor Integrated Technology, Institute of Semiconductors, \\ Chinese Academy of Sciences, Beijing 100083, China; zhenhuang@semi.ac.cn (Z.H.); \\ lizhaofeng@semi.ac.cn (Z.L.); dh0511@semi.ac.cn (H.D.); xdwang@semi.ac.cn (X.W.) \\ 2 Center of Materials Science and Opto-Electronics Engineering, University of Chinese Academy of Sciences, \\ Beijing 100049, China; fhyang@semi.ac.cn \\ 3 College of Materials Science and Opto-Electronics Technology, University of Chinese Academy of Sciences, \\ Beijing 100049, China \\ 4 School of Microelectronics, University of Chinese Academy of Sciences, Beijing 100049, China \\ 5 Department of Electronic Science and Technology, University of Science and Technology of China, \\ Hefei 230026, China \\ 6 State Key Laboratory for Superlattices and Microstructures, Institute of Semiconductors, Chinese Academy of \\ Sciences, Beijing 100083, China \\ 7 Beijing Academy of Quantum Information Science, Beijing 100193, China \\ 8 Beijing Engineering Research Center of Semiconductor Micro-Nano Integrated Technology, \\ Beijing 100083, China \\ * Correspondence: yanwei@semi.ac.cn
}

check for

updates

Citation: Huang, Z.; Yan, W.; Li, Z.; Dong, H.; Yang, F.; Wang, X. High-Responsivity, Low-Leakage Current, Ultra-Fast Terahertz Detectors Based on a GaN High-Electron-Mobility Transistor with Integrated Bowtie Antennas. Sensors 2022, 22, 933. https:// doi.org/10.3390/s22030933

Academic Editor: Qammer Hussain Abbasi

Received: 1 December 2021 Accepted: 23 January 2022 Published: 25 January 2022

Publisher's Note: MDPI stays neutral with regard to jurisdictional claims in published maps and institutional affiliations.

Copyright: (C) 2022 by the authors Licensee MDPI, Basel, Switzerland. This article is an open access article distributed under the terms and conditions of the Creative Commons Attribution (CC BY) license (https:// creativecommons.org/licenses/by/ $4.0 /)$.

\begin{abstract}
In this study, we fabricated three kinds of terahertz detectors with different leakage currents to analyze the plateau-like effect. The results indicate that the platform becomes increasingly apparent with the decrease in the leakage current. The fabricated device with the lowest leakage current shows a responsivity of $4.9 \mathrm{kV} / \mathrm{W}$ and noise equivalent power (NEP) of $72 \mathrm{pW} / \sqrt{\mathrm{Hz}}$. Further, it can be used for broadband detection between $215 \mathrm{GHz}$ and $232 \mathrm{GHz}$ with a voltage responsivity of more than $3.4 \mathrm{kV} / \mathrm{W}$, and the response time can be up to $8 \mathrm{~ns}$. Overall, the proposed device exhibits high sensitivity, large modulation frequency, and fast response, which indicates its excellent potential for detection and imaging applications in the $\mathrm{THz}$ range, including the detection of the $220 \mathrm{GHz}$ atmospheric window.
\end{abstract}

Keywords: terahertz detector; leakage currents; plateau-like effect; responsivity; broadband detection

\section{Introduction}

I recent years, terahertz $(\mathrm{THz})$ technology has been extensively applied in a variety of fields such as military applications, radio communication, climate monitoring, and medical imaging [1,2]. Consequently, the development of an ultra-fast and highly sensitive detector working in the sub- $\mathrm{THz}$ and $\mathrm{THz}$ regime has garnered significant research attention. Based on the nonlinear properties of two-dimensional electron gas (2DEG), field-effect transistors (FETs) have been verified as effective THz detectors. The FET-based THz (TeraFET) detectors have the advantages of room-temperature operation, high responsivity $\left(R_{V}\right)$, and low equivalent noise power (NEP) [3]. These detectors have been fabricated with different materials, including Si [4], graphene [5], GaAs [6], and InP [7]. Compared with the above materials, the high-electron mobility transistor (HEMT) based on a GaN heterojunction provides higher 2DEG channel density with unintentional doping, high breakdown voltage, and ultrafast response. All these properties make GaN HEMTs one of the most promising candidates for $\mathrm{THz}$ detection.

High responsivity, low-NEP, and high speed are crucial to realize the practical application of $\mathrm{THz}$ detectors. By introducing a planar antenna into $\mathrm{THz}$ detectors, the $\mathrm{THz}$ 
signals can be efficiently coupled and the sensitivity is improved [8]. Many antennas such as dipole [9], loop [10], and slot-spiral [11] have been utilized. As structures with linear polarization, strong directivity, and high $\mathrm{THz}$-wave coupling efficiency, bowtie antennas have been employed to fabricate high-performance detectors with optical responsivities $\left(R_{V}\right)$ of $2 \mathrm{~V} / \mathrm{W}$ [5], $220 \mathrm{~V} / \mathrm{W}$ [12], and $3.6 \mathrm{kV} / \mathrm{W}$ [13]. In addition, the excellent performance of the transistor is the basic premise to ensure the superior characteristics of $\mathrm{THz}$ detector, especially for the reduction in the leakage current of the transistor [14].

In this paper, we present an optimized GaN/AlGaN HEMT with a 150-nmong gate and an integrated bowtie antenna as an effective $\mathrm{THz}$ detector. Three kinds of devices with different leakage currents $\left(I_{L}\right)$ were fabricated by different technological processes. The results show that an infinitesimally small $I_{L}$ generates a plateau-like effect in the subthreshold region, resulting in a comparable output response near the threshold voltage $\left(V_{t h}\right)$. The detector with a smaller $I_{L}$ has a stronger photoresponse and a larger peak width. The fabricated device shows a high $R_{V}$ and low NEP. Furthermore, a stable output is maintained at the modulation frequency of $3.7 \mathrm{kHz}$, and the response time can reach $8 \mathrm{~ns}$. All of these properties indicate that our fabricated device is a potential $\mathrm{THz}$ detector for future applications.

\section{Detection Model and Characterization Setup}

The device model used to predict the THz $R_{V}$ and NEP consists of an HEMT and integrated bowtie antenna, as shown in Figure 1a. The fabricated HEMT has epilayers, including a 1.6- $\mu \mathrm{m}$ GaN buffer layer, a 1-nm AlN spacer, a 21-nm AlGaN barrier, and a 2-nm GaN cap layer. It was grown on a 2-inch sapphire substrate. A 2DEG layer was then generated on the top of GaN buffer. The SiN passivation layer was grown in three different ways: sample 1 was prepared by in situ growth through metal-organic chemical vapor deposition (MOCVD), sample 2 was grown by plasma-enhanced chemical vapor deposition (PECVD), and there was no passivation layer on sample 3. The samples grown in the three different ways generated different leakage currents, which were examined as follows.

(a)

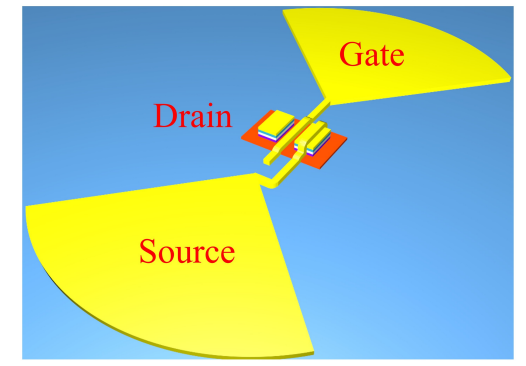

(c)

\begin{tabular}{|c|c|c|}
\hline \multirow{2}{*}{$\begin{array}{c}\text { Source } \\
\text { Antenna }\end{array}$} & Antenna & \multirow[b]{2}{*}{ Drain } \\
\hline & SiN Passivation Layer & \\
\hline $45 \mathrm{~nm} \mathrm{Au}$ & 2nm GaN Cap & $45 \mathrm{~nm} \mathrm{Au}$ \\
\hline $55 \mathrm{~nm} \mathrm{Ni}$ & $21 \mathrm{~nm}$ AlGaN Barrier & $55 \mathrm{~nm} \mathrm{Ni}$ \\
\hline $120 \mathrm{~nm} \mathrm{Al}$ & 1nm AlN Spacer & $120 \mathrm{~nm} \mathrm{Al}$ \\
\hline $20 \mathrm{~nm} \mathrm{Ti}$ & 2DEG Channel & $20 \mathrm{~nm} \mathrm{Ti}$ \\
\hline \multicolumn{3}{|c|}{$1.6 \mu \mathrm{m} \mathrm{GaN} \mathrm{Buffer}$} \\
\hline & Sapphire & \\
\hline
\end{tabular}

(b)

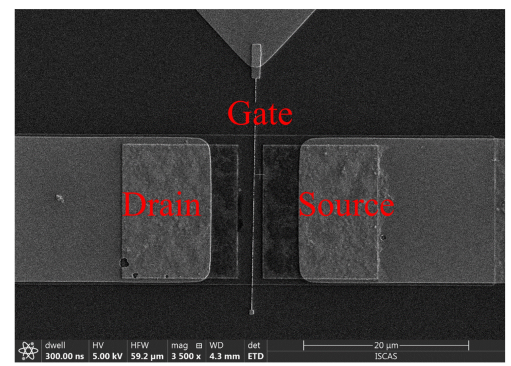

(d)

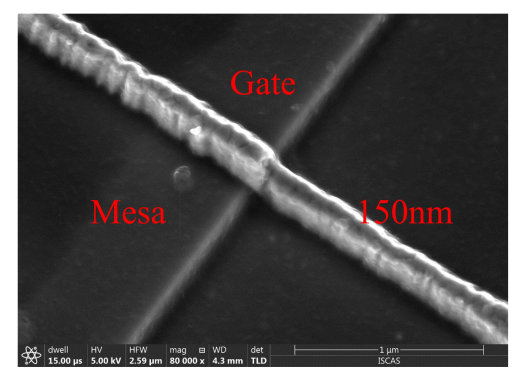

Figure 1. (a) Overview of the detection model. (b) Local diagram of $\mathrm{THz}$ detector at a magnification of $20 \mu \mathrm{m}$. (c) Specific parameters of HEMT. (d) Partial enlarged image of gate across the mesa at a magnification of $1 \mu \mathrm{m}$.

The device was prepared with the mesa isolation by inductively coupled plasma etching. Source and drain $(6 \mu \mathrm{m} \times 3 \mu \mathrm{m})$ with a gap of $3 \mu \mathrm{m}$ were aligned, and then e-beam deposition was used to form the metalayers: $\mathrm{Ti} / \mathrm{Al} / \mathrm{Ni} / \mathrm{Au}(20 / 120 / 55 / 45 \mathrm{~nm})$. This was followed by a rapid thermal annealing at $400 / 700 / 870{ }^{\circ} \mathrm{C}(170 / 40 / 30 \mathrm{~s})$ to form 
the source and drain ohmic contact, which provides an unimpeded channel for plasma waves. A bowtie antenna with an optimal radius of $60 \mu \mathrm{m}$ and radial size of 90 degrees was fabricated by lithography. Finally, a nanogate $(150 \mathrm{~nm})$ placed in the center of the gap between source and drain was aligned by electron beam lithography, and then magnetron sputtering was used to form Ni/Au (20/200 nm) metalayers. A schematic of the HEMT is shown in Figure 1c, and the finished device is presented in Figure 1b,d. The two arms of the bowtie antenna were integrated with the gate and source of the HEMT to form an asymmetric structure.

The experimental setup for the characterization of the detector is shown in Figure 2. Continuous-wave radiation was generated from a backward-wave oscillator (BWO) and focused onto the surface of the detector at the fundamental frequency (175-384 GHz) by two off-axis parabolic (OAP) mirrors (90 degree OAP, Effective Focalength (EFL) = $152.4 \mathrm{~mm}$ ). The received total power at every electromagnetic frequency was calibrated using a $\mathrm{THz}$ Golay cell. The detector was mounted on an FR-4 printed circuit board (PCB) and could be precisely moved by a motorized translation stage. The gate electrode was connected to a DC bias. The source was grounded, and the rectified $\mathrm{THz}$ voltage of the detector $\left(V_{D S}\right)$ was measured using a lock-in amplifier (Stanford Research Systems, SR830). A chopper (SR540, 4-3700 Hz) was used to convert the continuous wave into a square wave, and the modulation signal was simultaneously fed into the lock-in amplifier. A mixed signal oscilloscope (Agilent MSO7104B) was used to record the temporal response before the lock-in amplifier. The data were collected on a personal computer from a General Purpose Interface Bus (GPIB) line. The DC electrical performance of the detector was measured by a semiconductor device analyzer (Agilent B1500A).

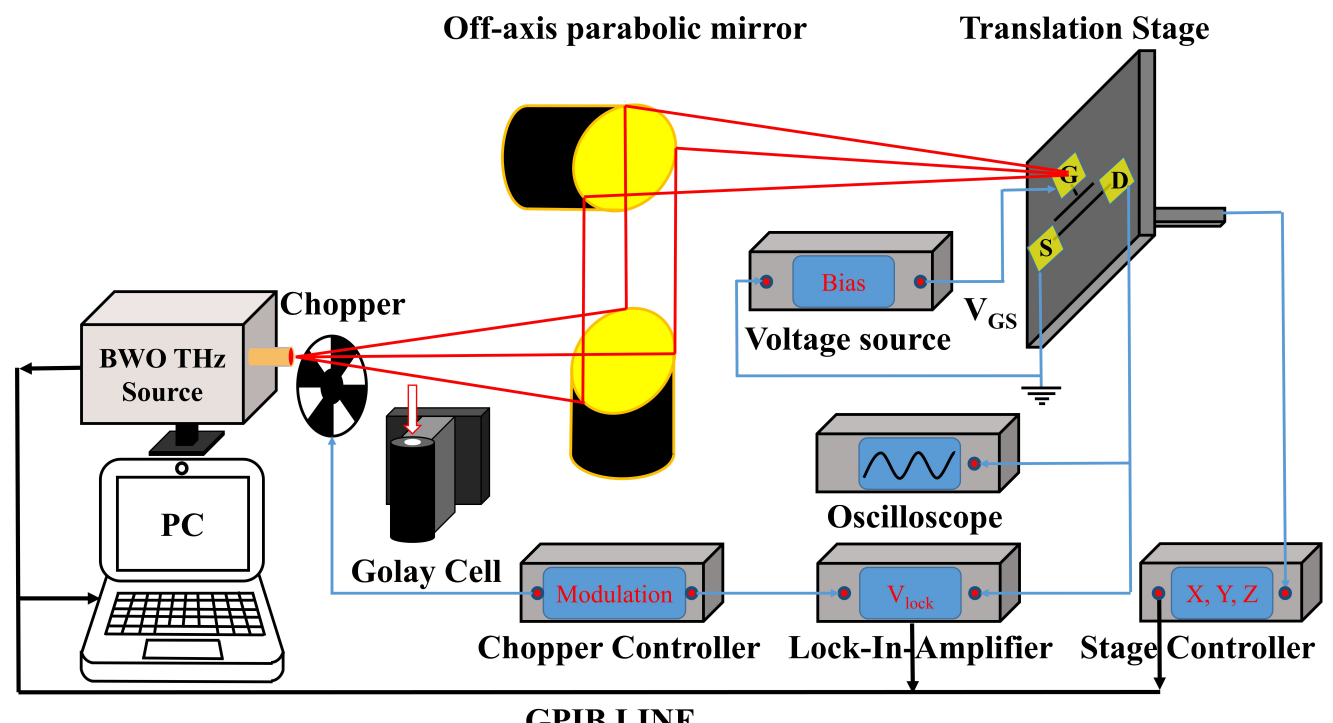

Figure 2. Experimental setup for the characterization of the $\mathrm{THz}$ detector.

\section{Results and Discussion}

The $R_{V}$ and NEP of the THz detectors are defined as follows:

$$
\begin{gathered}
R_{V}=\frac{S_{t} \cdot V_{D S}}{S_{\text {eff }} \cdot P_{t}} \\
V_{D S}=\frac{\pi}{\sqrt{2}} \cdot \frac{R_{\text {lock }}+R_{c h}}{R_{\text {lock }}} \cdot V_{\text {lock }} \\
\mathrm{NEP}=\frac{N_{V}}{R_{V}}=\frac{\sqrt{4 k T R_{c h}}}{R_{V}}
\end{gathered}
$$


where $S_{\text {eff }}$ is the active area of the THz detector, $S_{t}$ is the beam spot size, and $P_{t}$ is the total power of the THz source. The term $\left(R_{\text {lock }}+R_{c h}\right) / R_{\text {lock }}$ represents the loading effect, which causes the $R_{V}$ drop after the gate voltage $\left(V_{G S}\right)$ to be less than the $V_{\text {th }}$ measured using the lock-in amplifier [15]. $R_{\text {lock }}=10 \mathrm{M} \Omega$ is the input impedance of the lock-in amplifier, and $V_{\text {lock }}$ is the output voltage, which is directly read out from the amplifier. Since the detected signal is a square wave, a factor of $\pi / \sqrt{2}$ must be included in the calculation. In the equation of NEP, $N_{V}$ is the thermal noise of the HEMT, $\mathrm{k}$ is the Boltzmann constant, T is the absolute temperature (room temperature $=300 \mathrm{~K}$ ), and $R_{c h}$ is the channel resistance of the HEMT.

Figure 3 shows the DC output, $G_{0}$, and $I_{L}$ characteristics of the GaN/AlGaN HEMT with the integrated bowtie antenna. According to Figure $3 \mathrm{a}$, the $V_{t h}$ of the fabricated device is around $-4.5 \mathrm{~V}$, and the value of $R_{c h}$ at $-4.5 \mathrm{~V}$ is $15.2724 \mathrm{M} \Omega$ from Figure $3 \mathrm{~d}$. The $I_{L}$ corresponding to samples 1,2 , and 3 are $5 \times 10^{-8} \mathrm{~A}, 3 \times 10^{-7} \mathrm{~A}$, and $9 \times 10^{-4} \mathrm{~A}$, respectively, from Figure 3c.

(a)

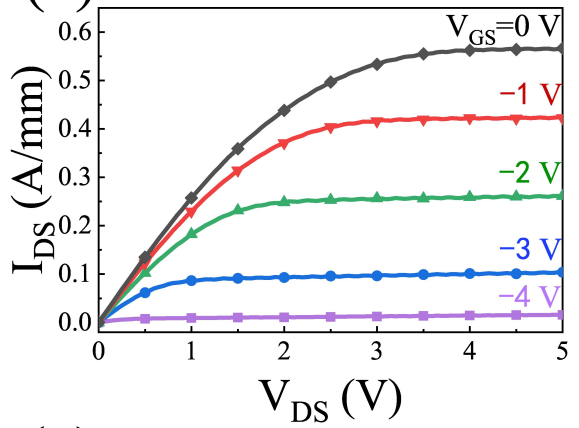

(c)

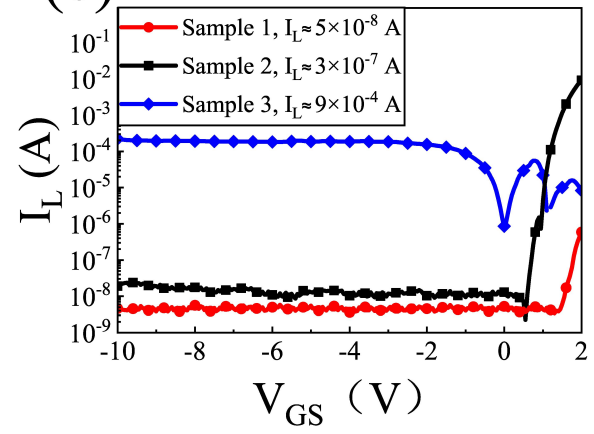

(b)

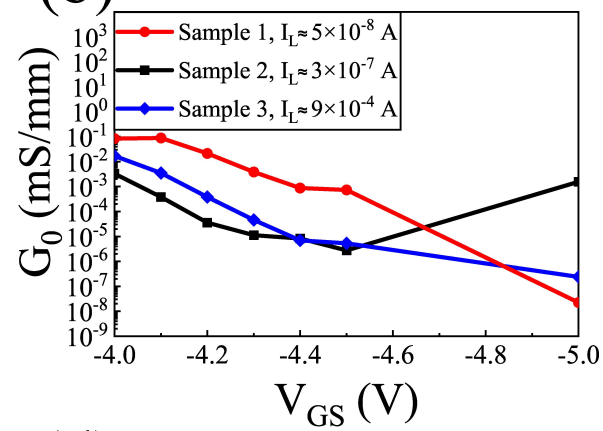

(d)

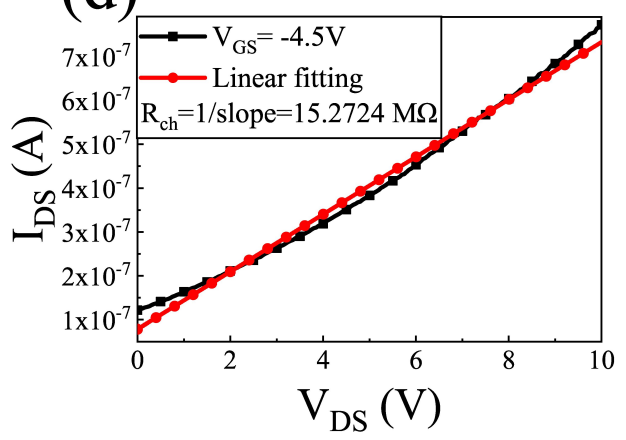

Figure 3. (a) I-V characteristics of sample 1. (b) Measured source-drain conductance $\left(G_{0}\right)$ as a function of the $V_{G S}$ in the subthreshold regime. (c) Different leakage currents of the three samples. (d) Output characteristics of sample 1 at $V_{G S}=-4.5 \mathrm{~V}$.

By scanning the $\mathrm{THz}$ detector through the translation stage, $S_{t}$ was obtained in a range tuned from $19.63 \mathrm{~mm}^{2}$ up to $50.27 \mathrm{~mm}^{2}$ (the radius of the spot is from $2.5 \mathrm{~mm}$ to $4 \mathrm{~mm}$ ) with the frequency ranging from 175 to $384 \mathrm{GHz}$. Further, the effective area $S_{\text {eff }}$ represented the active antenna dimensions of $\pi \times(60 \mu \mathrm{m})^{2} \times 0.5$. The total power $P_{t}$ was calibrated by a standard Golay cell. All the related parameters were substituted into Equations (1) and (3) to obtain the $R_{V}$ with and without the bowtie antenna from 175 to $384 \mathrm{GHz}$. The results are shown in Figure 4.

Figure $4 \mathrm{a}, \mathrm{b}$ indicate that the resonant frequency was $240 \mathrm{GHz}$, and the impedance was (50-j25) $\Omega$ at this point, which met with the design requirement. It is clear from Figure $4 \mathrm{~d}$ that the maximum $R_{V}$ was $4.9 \mathrm{kV} / \mathrm{W}$ at $227 \mathrm{GHz}$, which is among the best values reported for nonresonant detection by GaN/AlGaN HEMTs integrated with bowtie antennas at room temperature. Furthermore, the simulation results are in excellent agreement with the measured results. The shift in frequency is due to the increased antenna size of the 
experimentally fabricated device, with the use of a positive photoresist in the lithography process, and the difference in $R_{V}$ may be attributed to the fact that perfect impedance matching cannot be achieved between the antenna and the HEMT in the fabrication process. The $R_{V}$ could be further improved by optimizing the substrate thickness [16]. Further, the minimum NEP was $0.1 \mathrm{nW} / \sqrt{\mathrm{Hz}}$ of $V_{G S}=-4.5 \mathrm{~V}$. Compared to the device without the bowtie antenna, the $R_{V}$ of the device with integrated bowtie antenna was significantly improved, which is mainly due to the optimized nanogate, proper antenna size, and the asymmetric structure.

(a)

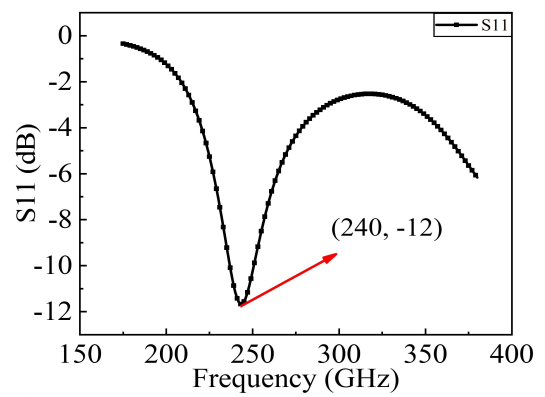

(c)

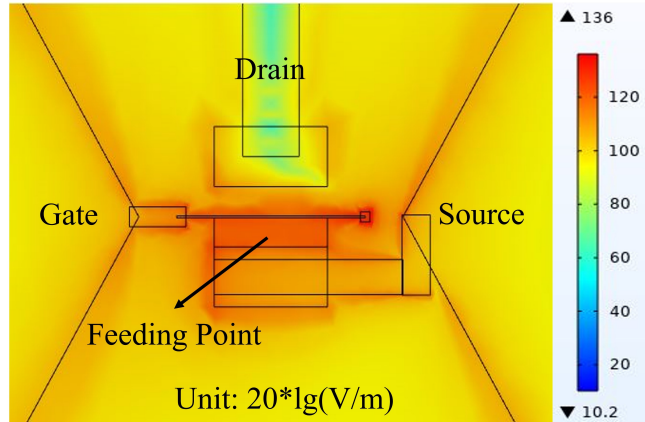

(b)

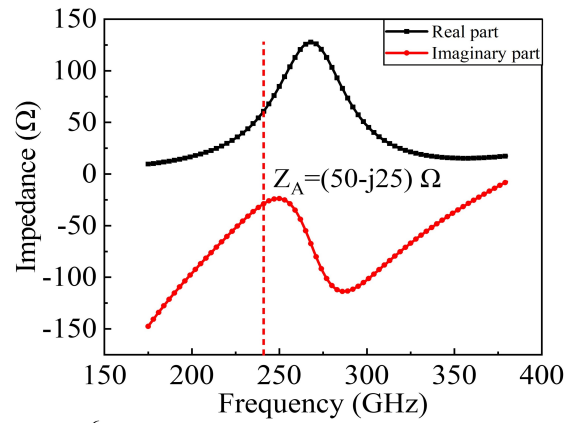

(d)

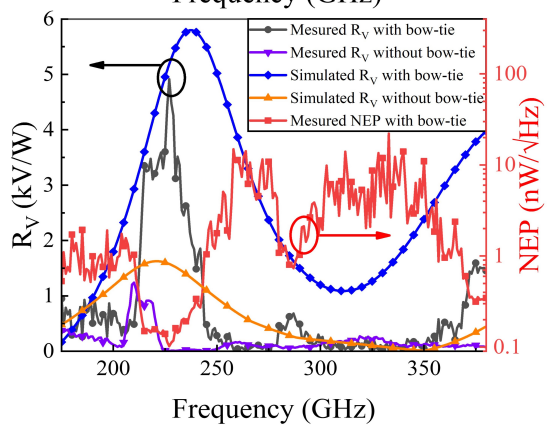

Figure 4. (a) The simulated S11 of the structure. (b) The simulated impedance of the structure. (c) Distribution of the electric field on the surface of the TeraFET detector around the gate (top view). (d) Measured and simulated $R_{V}$ with and without a bowtie antenna from 175 to $384 \mathrm{GHz}$. The red line indicates the measured NEP of the detector with a bowtie antenna. All the results above correspond to sample 1 .

The asymmetric structure results in an asymmetrical distribution of the electric field, which leads to a potential difference between the source and drain [13]. In the FETbased terahertz detector system, the whole modulation process takes place in the channel, especially in the region directly regulated by the gate. The extremely short gate can localize the coupled $\mathrm{THz}$ energy within a very narrow region, thereby significantly enhancing the modulation of the plasma wave and improving the sensitivity of the detector [8]. This phenomenon is clearly shown in Figure 4c, which was obtained by the commercial software COMSOL; all the related parameters in the simulation were the same as the reality in Figure 1c, and the feeding point of the antenna is shown as an illustration. A lumped port excitation was set between the source and gate electrode, and then the PML (Perfect Matchayer) boundary condition wraps the model inside. In the actual measurement, the characteristics of sample 2 and 3 versus the frequency were in the same variation tendency as sample 1 , the differences between them were the maximum responsivity and minimum NEP. Their characteristics versus the $V_{G S}$ were more intuitive, as shown in the following sections.

Figure 5a shows the $V_{D S}$ versus $V_{G S}$ and $V_{\text {lock }}$ curves of the three samples with different $I_{L}$. For nonresonant detection, $I_{L}$ is a vital parameter to derive the detector output. $I_{L}$ suppresses the detector response in the subthreshold region, leading to a maximum value 
in the photoresponse versus the gate voltage. For the same type of HEMTs, the width of the peak can be theoretically calculated [14] as follows:

$$
W_{\text {peak }} \propto(1 / \epsilon)
$$

where $\ln (1 / \epsilon)$ is a dimensionless parameter, which is a slowly varying function of $I_{L}$. In principle, the smaller the $I_{L}$, the larger the value of $\ln (1 / \epsilon)$, and the larger the peak width. However, from the perspective of the loading effect, this phenomenon occurs because $G_{0}$ (in Figure $3 \mathrm{~b}$ ) kept decreasing when the gate voltage was below the $V_{t h}$ in the low $I_{L}$ state; thus, the loading effect became more important according to Equation (2). From the inset picture of Figure $5 \mathrm{a}, V_{\text {lock }}$ kept a nearly flat state between $-4.4 \mathrm{~V}$ to $-4.6 \mathrm{~V}$ even though the $I_{L}$ changed. However, the variation tendency of $G_{0}$ in the subthreshold region varied with the $I_{L}$ (in Figure $3 \mathrm{~b}$ ). For the smallest $I_{L}$ of sample $1, G_{0}$ would keep decreasing when the gate voltage was below the $V_{\text {th }}$; thus, $V_{D S}$ would broaden the peak width between $-4.36 \mathrm{~V}$ to $-4.8 \mathrm{~V}$, as sample 2 of $-4.39 \mathrm{~V}$ to $-4.7 \mathrm{~V}$. The more obvious the reduction trend, the wider the platform. On the contrary, for the larger $I_{L}$ of sample $3, G_{0}$ increased gradually below the $V_{t h}$; in this case, the peak width decreased between $-4.39 \mathrm{~V}$ to $-4.5 \mathrm{~V}$. Furthermore, the measured output increased with the decrease in the leakage current due to the excellent gate-control ability. Further, we also simulated the normalized $V_{D S}$ of different leakage currents, and the result agreed well with the actual situation. The calculation and derivation of the simulation came from the literature [14], and the specific parameters required for the simulation are described in this paper.

The variation trends of $R_{V}$ and NEP as a function of the gate voltage at $227 \mathrm{GHz}$ for sample 1 are presented in Figure $5 \mathrm{c}$. The maximum $R_{V}$ was $4.9 \mathrm{kV} / \mathrm{W}$ of $-4.5 \mathrm{~V}$, and the minimum NEP was $72 \mathrm{pW} / \sqrt{\mathrm{Hz}}$ of $-4.7 \mathrm{~V}$. It can be seen in Figure $5 \mathrm{~d}$ that the photovoltage remained almost constant in the modulation frequency range of 4-3700 Hz, which was limited by the chopper. The $V_{\text {lock }}$ showed noise (at multiples of $50 \mathrm{~Hz}$ ) due to power line interference. In this case, large noise can be generated when using lock-in technology. The temporal response of the fabricated device was determined when the incident light was chopped at $1 \mathrm{kHz}$, and the readout voltage was recorded by a mixed signal oscilloscope. The response time of this device is characterized by measuring the time between $10 \%$ and $90 \%$ of the generated signal, either on the rising or the falling edge [17]. For the measurement of the transient response of the photovoltage, the output port of device was directly connected with the oscilloscope, the lock-in amplifier was not operating at this time. The chopper continued to work, the chopping frequency was $1 \mathrm{kHz}$, and then the continuous wave converted into the square wave. The oscilloscope displayed the operating status of the device with and without terahertz, and the time of device from on-state to off-state was the transient response time. It is worth noting that the output voltage of the device was around $1.6 \mathrm{mV}$ without the preamplifier, which was larger than the noise generated by devices and the environment. Thus, we could directly read out the output voltage without the lock-in amplifier. There is no denying the fact that the readout voltage response by the oscilloscope was not accurate, but the transient response time was not affected by the noise. The results are shown in Figure 5e,f. The minimum response time of the TeraFET detector was calculated as [18]

$$
t_{\min } \propto \frac{L^{2}}{\mu V_{G S}} \approx 1.7 \times 10^{-10} \mathrm{~s}
$$

Here, $L$ is the gate-controlled length (nearly $1 \mu \mathrm{m}$ from Figure $4 \mathrm{a}$ ), and $\mu$ is the electron mobility $\left(1300 \mathrm{~cm}^{2} / \mathrm{Vs}\right)$. The response time of the fabricated device was $8 \mathrm{~ns}$, which is inferior to the theoretical value due to the bandwidth limitation of the oscilloscope.

Under the excitation of the THz electric field, 2DEG with high mobility could complete the signal transmission between source and drain at an extremely high speed. This process was much faster than that in a thermoelectric detector with an electron temperature gradient formed by the electron-electron and electron-phonon interaction $[19,20]$ and in 
a photodetector based on photo-assisted tunneling [21]. The short response time is an important parameter to define the merit of a $\mathrm{THz}$ detector, which is usually desired in high-performance devices. The performance of the fabricated $\mathrm{THz}$ detector is comparable or even superior to previously reported detectors (see Table 1).

(a)
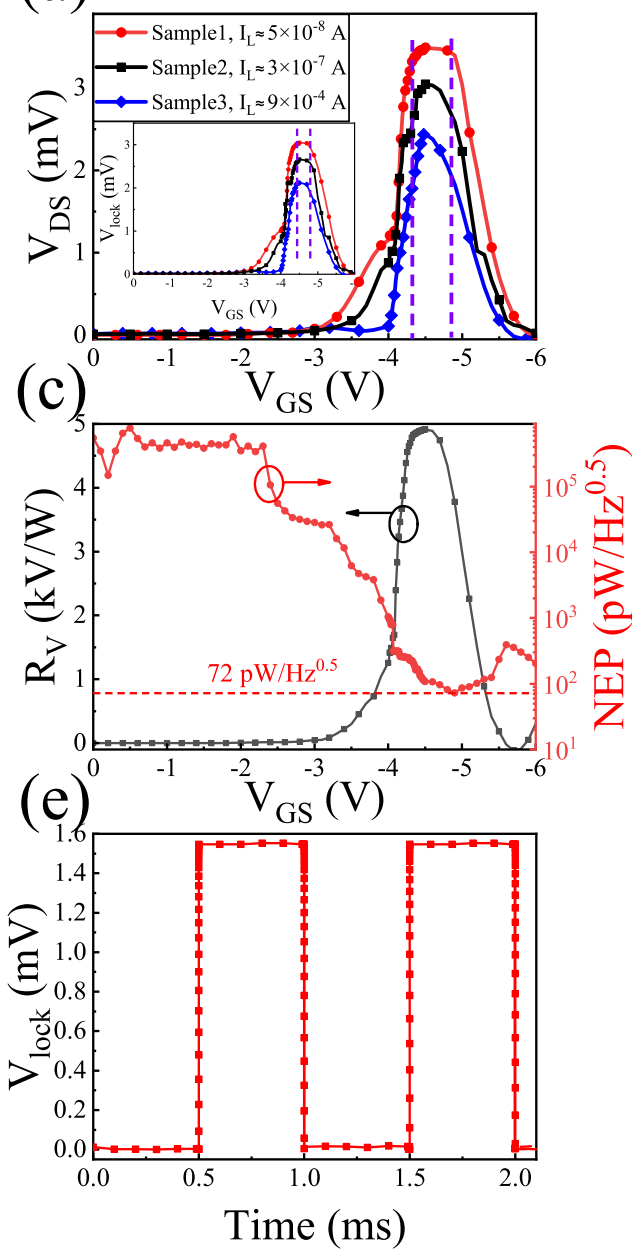

(b)
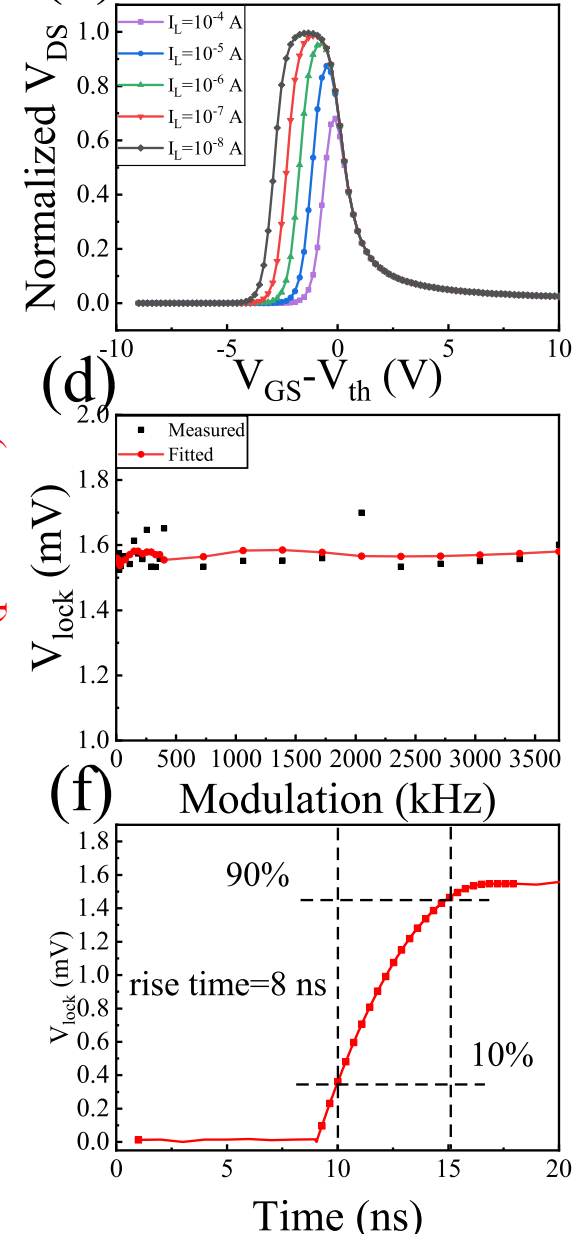

Figure 5. (a) Measured $V_{D S}$ versus $V_{G S}$ and $V_{\text {lock }}$ curves of the three samples with different $I_{L}$. (b) Simulated normalized $V_{D S}$ as a function of the $V_{G S}$ at different leakage currents. (c) Responsivity and NEP versus $V_{G S}$ of sample 1. (d) Measured and fitted $V_{\text {lock }}$ versus amplitude modulation frequency of electromagnetic wave of sample 1. (e) Temporal response of the fabricated device chopped by an electronic modulator at $1 \mathrm{kHz}$ of sample 1 . (f) Measured response time of sample 1.

Table 1. Comparison of the performance and efficiency of the proposed and previously reported detectors at room temperature.

\begin{tabular}{ccccc}
\hline Architecture & NEP $(\mathbf{p W} / \sqrt{\mathbf{H z}})$ & $\boldsymbol{R}_{\boldsymbol{V}}(\mathbf{V} / \mathbf{W})$ & Response Time (ns) & Measurement \\
\hline This paper & 72 & 4900 & 8 & Lock-in + Oscilloscope \\
GaN bowtie [13] & 40 & 3600 & - & Lock-in \\
Si bowtie [12] & 48 & 220 & - & Preamplifier + Lock-in \\
GaN bowtie [22] & 25 & - & - & Lock-in \\
Graphene spiral [17] & 350 & 28 & 9000 & Preamplifier + Lock-in + Oscilloscope \\
Si patch [23] & 31 & - & 0.012 & Autocorrelation measurement \\
SiGe patch [24] & - & 15 & 1.7 & On-chip amplifier \\
\hline
\end{tabular}




\section{Conclusions}

In conclusion, we fabricated three kinds of $\mathrm{THz}$ detectors with different passivation technologies and analyzed the effect of the leakage current on the device performance. The results revealed the generation of a plateau-like effect, and a higher photoresponse was obtained at a lower leakage current. A high sensitivity of $4.9 \mathrm{kV} / \mathrm{W}$ and $72 \mathrm{pW} / \sqrt{\mathrm{Hz}}$ with a low leakage current was achieved by integration with a bowtie antenna, and the response time was up to $8 \mathrm{~ns}$. Furthermore, the device response was superior to that of the existing TeraFET detectors. In addition, the NEP of our fabricated device has improved close to that of the widely-used Schottky diodes detectors [25] and TeraFET detectors and could show excellent characteristics in room temperature. Overall, based on its unique attributes of room-temperature operation, low leakage current, and ultra-fast response, this detector is a promising candidate for future $\mathrm{THz}$ applications.

Author Contributions: Conceptualization, Z.H.; methodology, Z.H.; software, Z.H.; formal analysis, Z.H. and W.Y.; investigation, Z.H. and H.D.; data curation, Z.H. and W.Y.; writing-original draft preparation, Z.H.; writing-review and editing, W.Y., X.W. and Z.L.; supervision, F.Y. and X.W.; funding acquisition, Z.L. and W.Y. All authors have read and agreed to the published version of the manuscript.

Funding: This work was partly supported by tge National Key R\&D Program of China under Grant 2018YFE0204000 and the National Natural Science Foundation of China (Grant No. 61971395).

Data Availability Statement: The data that support the findings of this study are available from the corresponding author upon request.

Conflicts of Interest: The authors declare no conflict of interest.

\section{References}

1. Ergun, S.; Sonmez, S. Terahertz technology for military applications. J. Manag. Inf. Sci. 2015, 3, 13-16. [CrossRef]

2. Kürner, T. Towards future THz communications systems. Terahertz Sci. Technol. 2012, 5, 11-17.

3. Dyakonov, M.; Shur, M. Detection, mixing, and frequency multiplication of terahertz radiation by two-dimensional electronic fluid. IEEE Trans. Electron Devices 1996, 43, 380-387. [CrossRef]

4. Knap, W.; Teppe, F.; Meziani, Y.; Dyakonova, N.; Lusakowski, J.; Boeuf, F.; Skotnicki, T.; Maude, D.; Rumyantsev, S.; Shur, M. Plasma wave detection of sub-terahertz and terahertz radiation by silicon field-effect transistors. Appl. Phys. Lett. 2004, 85, 675-677. [CrossRef]

5. Yang, X.; Vorobiev, A.; Generalov, A.; Andersson, M.A.; Stake, J. A flexible graphene terahertz detector. Appl. Phys. Lett. 2017, 111, 021102. [CrossRef]

6. Popov, V.; Ermolaev, D.; Maremyanin, K.; Maleev, N.; Zemlyakov, V.; Gavrilenko, V.; Shapoval, S.Y. High-responsivity terahertz detection by on-chip InGaAs/GaAs field-effect-transistor array. Appl. Phys. Lett. 2011, 98, 153504. [CrossRef]

7. Kurita, Y.; Ducournau, G.; Coquillat, D.; Satou, A.; Kobayashi, K.; Boubanga Tombet, S.; Meziani, Y.; Popov, V.; Knap, W.; Suemitsu, T.; et al. Ultrahigh sensitive sub-terahertz detection by InP-based asymmetric dual-grating-gate high-electron-mobility transistors and their broadband characteristics. Appl. Phys. Lett. 2014, 104, 251114. [CrossRef]

8. Sun, J.; Qin, H.; Lewis, R.; Sun, Y.; Zhang, X.; Cai, Y.; Wu, D.; Zhang, B. Probing and modelling the localized self-mixing in a GaN/AlGaN field-effect terahertz detector. Appl. Phys. Lett. 2012, 100, 173513.

9. Tanigawa, T.; Onishi, T.; Takigawa, S.; Otsuji, T. Enhanced responsivity in a novel AlGaN/GaN plasmon-resonant terahertz detector using gate-dipole antenna with parasitic elements. In Proceedings of the IEEE 68th Device Research Conference, Notre Dame, IN, USA, 21-23 June 2010; pp. 167-168.

10. Labidi, M.; Choubani, F. Performances enhancement of metamaterial loop antenna for terahertz applications. Opt. Mater. 2018, 82, 116-122. [CrossRef]

11. Huang, Z.; Li, Z.; Dong, H.; Yang, F.; Yan, W.; Wang, X. Novel Broadband Slot-Spiral Antenna for Terahertz Applications. Photonics 2021, 8, 123. [CrossRef]

12. Ikamas, K.; Čibiraitè, D.; Lisauskas, A.; Bauer, M.; Krozer, V.; Roskos, H.G. Broadband terahertz power detectors based on 90-nm silicon CMOS transistors with flat responsivity up to 2.2 THz. IEEE Electron Device Lett. 2018, 39, 1413-1416. [CrossRef]

13. Sun, J.; Sun, Y.; Wu, D.; Cai, Y.; Qin, H.; Zhang, B. High-responsivity, low-noise, room-temperature, self-mixing terahertz detector realized using floating antennas on a GaN-based field-effect transistor. Appl. Phys. Lett. 2012, 100, 013506.

14. Knap, W.; Kachorovskii, V.; Deng, Y.; Rumyantsev, S.; Lü, J.Q.; Gaska, R.; Shur, M.; Simin, G.; Hu, X.; Khan, M.A.; et al. Nonresonant detection of terahertz radiation in field effect transistors. J. Appl. Phys. 2002, 91, 9346-9353. [CrossRef]

15. Hou, H.; Liu, Z.; Teng, J.; Palacios, T.; Chua, S.J. A sub-terahertz broadband detector based on a GaN high-electron-mobility transistor with nanoantennas. Appl. Phys. Express 2016, 10, 014101. [CrossRef] 
16. Zhang, B.; Yan, W.; Li, Z.; Bai, L.; Yang, F. Analysis of substrate effect in field effect transistor terahertz detectors. IEEE J. Sel. Top. Quantum Electron. 2016, 23, 1-7. [CrossRef]

17. Guo, W.; Wang, L.; Chen, X.; Liu, C.; Tang, W.; Guo, C.; Wang, J.; Lu, W. Graphene-based broadband terahertz detector integrated with a square-spiral antenna. Opt. Lett. 2018, 43, 1647-1650. [CrossRef]

18. Kachorovskii, V.Y.; Shur, M. Field effect transistor as ultrafast detector of modulated terahertz radiation. Solid-State Electron. 2008, 52, 182-185. [CrossRef]

19. Sun, D.; Aivazian, G.; Jones, A.M.; Ross, J.S.; Yao, W.; Cobden, D.; Xu, X. Ultrafast hot-carrier-dominated photocurrent in graphene. Nat. Nanotechnol. 2012, 7, 114-118. [CrossRef]

20. Somphonsane, R.; Ramamoorthy, H.; Bohra, G.; He, G.; Ferry, D.; Ochiai, Y.; Aoki, N.; Bird, J. Fast energy relaxation of hot carriers near the dirac point of graphene. Nano Lett. 2013, 13, 4305-4310. [CrossRef]

21. Ariyoshi, S.; Otani, C.; Dobroiu, A.; Sato, H.; Kawase, K.; Shimizu, H.; Taino, T.; Matsuo, H. Terahertz imaging with a direct detector based on superconducting tunnel junctions. Appl. Phys. Lett. 2006, 88, 203503. [CrossRef]

22. Bauer, M.; Rämer, A.; Chevtchenko, S.A.; Osipov, K.Y.; Čibiraitè, D.; Pralgauskaite, S.; Ikamas, K.; Lisauskas, A.; Heinrich, W.; Krozer, V.; et al. A high-sensitivity AlGaN/GaN HEMT terahertz detector with integrated broadband bow-tie antenna. IEEE Trans. Terahertz Sci. Technol. 2019, 9, 430-444. [CrossRef]

23. Lisauskas, A.; Ikamas, K.; Massabeau, S.; Bauer, M.; Čibiraitè, D.; Matukas, J.; Mangeney, J.; Mittendorff, M.; Winnerl, S.; Krozer, V.; et al. Field-effect transistors as electrically controllable nonlinear rectifiers for the characterization of terahertz pulses. APL Photonics 2018, 3, 051705. [CrossRef]

24. Wu, K.; Ducournau, G.; Hella, M.M. A $240 \mathrm{GHz}$ receiver with $6 \mathrm{~Gb} / \mathrm{s}$ data rate based on plasma wave detection in SiGe technology. In Proceedings of the 2019 IEEE 44th International Conference on Infrared, Millimeter, and Terahertz Waves (IRMMW-THz), Paris, France, 1-6 September 2019; pp. 1-2.

25. Sakhno, M.; Golenkov, A.; Sizov, F. Uncooled detector challenges: Millimeter-wave and terahertzong channel field effect transistor and Schottky barrier diode detectors. J. Appl. Phys. 2013, 114, 164503. [CrossRef] 